\title{
Community health priorities: Lessons
prevention from Balaka district, Malawi
} Warren Parker ${ }^{1}$, Thaddeus Pennas ${ }^{2}$, Innocent Kommwa ${ }^{3}$

1. Independent Consultant, Cape Town, South Africa

2. FHI360, Washington D.C., USA

3. Health Communication for Life (HC4L), Lilongwe, Malawi

Date Received: 04-Oct-2017

Revision Received: 18-Dec-2017

Date Accepted: 06-Jan-2018

Correspondence:

Warren Parker

(warrenmparker@mac.com)

\section{Introduction}

\section{Abstract}

Communication to address priority health challenges typically draws on epidemiological research in conjunction with referencing global and country strategies. While community-level perspectives on health challenges typically align with national priorities, nuances and barriers that constrain health response may be less well known. To deepen understanding of the relation between situational aspects of health and ways of making meaning, community insights into health priorities were explored.

\section{Methods}

Action Media, an established participatory research methodology for informing health communication design and strategy, was undertaken with parents of children under 5 in Balaka District, Malawi.

\section{Results}

Participants identified malaria as a priority concern in their community. While it was reported that there was strong commitment to consistent use of long lasting insecticide treated nets, there was frustration that malaria incidence remained high. It was observed that risk of exposure to mosquito bites was increased as a result of chores and other activities that extended into the early evenings prior to retiring to bed under a bed net. Bed bug infestations were said to be a common and ongoing concern. Some community members alleviated discomfort by abandoning their beds and sleeping outside without adequate protection while others boiled their bed nets to remove bed bugs. Accessing distant health facilities when ill with malaria was difficult for adults and children. Participants identified the need for effective strategies to address these concerns including accessing mosquito repellant, eradicating bed bugs, treating malaria locally and collaborating in malaria control activities.

Conclusions

Meeting targets for malaria prevention requires consideration of contextual factors that undermine effective malaria prevention in affected communities. Such factors are not immediately apparent through epidemiological data. Regularly assessing contextual challenges in high malaria incidence areas provides opportunities to understand gaps, to refine intervention strategies and to inform communication programming.

\section{Introduction}

Exposure to ill health at a population level is determined by a range of circumstances including vectors of disease, geographic locale, living circumstances and capacity to address risk. Addressing risk includes consideration of the relation between knowledge, context and culture towards support for individual or collective action ${ }^{1}$. Communication campaigns and activities to promote health employ diverse strategies. These include individual-focused approaches that draw on biomedical frameworks and rationalist assumptions about health behavior, approaches that emphasize social learning through role modeling and diffusion of technologies or concepts, and approaches that consider audience participation and action towards social mobilization ${ }^{2,3,4}$. Success of communication interventions depends on sound understanding of health challenges of audiences in conjunction with assessing the reception environment with regard to behavioral and socio-cultural concerns.

There is evidence to suggest that health communication is strengthened by approaches that explore health challenges in conjunction with participatory engagement activities to address understanding of health at community level. Participatory approaches consider health vulnerabilities in context, and take into account the potential for audiences to move from being recipients of information to empowered actors capable of dialogue, reflection and action in relation to their own health. To support understanding of health priorities at community level, a formative participatory study was undertaken with parents of children under 5 in the Balaka district of Malawi.

\section{Methods}

Action Media is an established methodology for health communication that derives insightinto audienceperspectives, fosters reflection and problem-solving around health issues, and informs design of communication strategies and interventions. Action Media provides an alternative to top-down 'targeted' approaches that may not adequately consider the extent of health knowledge, the dimensions of the enabling context for health, or the specific health challenges and capabilities of intended audiences ${ }^{6}$. Working with small groups' representative of broader audience characteristics, Action Media findings have been applied to inform diverse national and regional health communication products, activities, strategies and campaigns around the world. Employing a multi-session engagement process, the approach goes beyond conventional qualitative techniques such as focus group discussions. Relevance of findings to culturally and contextually similar audiences emerges as a product of the deeper exploration of health challenges in relation to locale, cultural meanings attached to health, and strengthening of commitment to health promotive action through communication and other support. 
Action Media was utilized to support the Malawi Ministry of In small group discussions, it was noted that it was likely that Health's Moyo ndi Mpamba (Life is Precious) public awareness - people were being bitten by mosquitos in the early evening campaign. The research team included representatives from prior to going to bed, and therefore prior to LLIN use. This the Ministry of Health and non-governmental organizations point was reinforced during the community visit, where active in health communication. Balaka District was selected on the basis of being generally representative of similar districts in the south of Malawi that have predominantly rural populations and that face a range of similar health challenges. Community participants were selected from the rural area surrounding Namikombe Primary School with the consent of the local Chief. The participant group comprised 14 men and women aged from 20 to 35 years. All were parents of children under 5 and at least half of the participants had two or more children. All were rural farmers. As this was a formative study to inform programme design, ethical review was not mandatory. Nonetheless, the study was guided by a research protocol that included an ethics component consistent with international guidelines. School classrooms were utilized as a venue for discussion and collaborative engagement took place over four days. At the outset, participants were provided with information on the planned activities and informed written consent was required. Activities to foster trust and open communication followed. Participants initially explored community values and health concerns in general, and then undertook participatory activities including large group discussions and independent small group work to unpack emerging issues and to deepen understanding of potential solutions. Linguistic, aesthetic and cultural perspectives were integrated through identifying locally relevant slogans and idioms, as well a developing songs, dance and artwork to support culturally meaningful communication. Researchers also conducted
brief community visit to verify findings emerging from the brief community visit
participatory sessions.

\section{Results}

Participants provided insight into their daily activities, noting that peaceful community and family life brought happiness with communal activities such as traditional dance, gospe music, watching football and community banking bein members brought hardship. Three priority health issues were identified - long distances to health facilities, lack of potable water and malaria. Malaria was discussed in detail potable findings are presented below.

\section{Knowledge about malaria}

Participants demonstrated accurate knowledge of malaria including symptoms and control measures. It was reported that children and adults in the community fell ill regularly with malaria despite the acknowledged relevance an widespread and committed use of Long Lasting Insecticidal Nets (LLINs) by community members.

\section{Exposure to mosquito bites}

The contradiction between perceived high levels of malaria incidence and widespread bed net use was attributed to exposure to mosquitos before going to bed, disruption of
LLIN use as a result of infestations of bed bugs, and belief that the chemicals for treating nets were neither and belief nor long-acting and that mosquito might be able to pass nor long-acting, and that mosquito might be able to pass hat preve of exposure to malaria was not guran the priction alternative options. male community member observed:

We suspect it is the mosquito bites we get before we go to bed. We hee chatting as a community in the early evening and we might have If supper late due to chores or [coming back late from] barvesting. Community visit, Day 3).

\section{Bed bug infestations}

Bed bug infestations had been ongoing in the community over the past four years and although the matter had been taised with the local chief, no concrete solution had emerged. Apart from discomfort from bites, bed bugs could be seen the chemicals on LIINs did not have any effect on these insects. Some community members were boiling their bed nets to kill the bed bugs, while others abandoned their bedding and slept outside without the benefit of an UIN

\section{Malaria prevention and treatment}

was noted that there was a lack of community cong and that such omissions increased the risks or malaria for

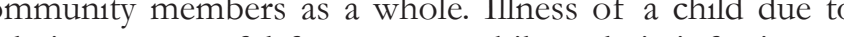
(alatia was stressful for parents, while malaria infection of parte health sevice access, constrained farming activities and had hegative impacts on household, community and national development.

Long distances to health facilities, transport costs and envistoris flooding discouraged access to treatment and resulted in a situation where "you can even die on the way to the clinic" (Participant, Male Group, Session

, Day 1 )

\section{Potentials for action}

There was frustration that there did not appear to be anything that could immediately be done to address the factors identified as increasing malaria risk, even though the government recognized that malaria was a kille LIIN discussions and large proun feedback sessions small group Chesions.

- Chemicals used on LLINs should be improved.

- Insect repellant should be made available to preven -

- Chemicals to eradicate bed bugs should be made available. - Health Surveillance Assistants (HSAs) should be allowed to minister malaria treatment in the community rather than requiring persons ill with malaria to travel to distant health facilities.

- Community members should work together to clear swampy areas and all should be responsible for ensuring that pools of stagnant water around households and in the

Drawing in village headmen and the
Critical reflection emerging through the Action Media proces is in that moment wue get meloria" (Male community member childcare, increased expenses due to costs of transport fo

\section{Malawi Medical Journal (2);99-102 June 2018}

\section{Supportive communication} prompted interest in obtaining communal benefits through
collective action. Such concepts framed the development of ideas for communication to support malaria prevention and treatment. For example, songs and poems were developed by participants that included verses imploring community members to seek treatment for maria emphasizing the importance of following prescribed dosages for malarin

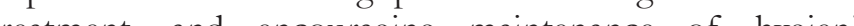
households and community environment.

Proverbs in the local language (Chichewa) wee put forwad. Proverbs in the local language (Chichewa) were put forward. For example, "Pali chabwino paliso choyipd" (meaning that good dwe the malaria, illustrates that although mosquito nets are a good buos), the "Chasmua a bugs), and "Chosamu anabphilkina mimasamb" (meaning he arrogant one was cooked together with the leaves highlighting that people should talk to and cooperate with tackle issues of mutual interest and not waiting for solutions to come from the outside: "We have realived that now" there are atportunities for us to solve some of these problems ourselues" (Fema participant, Session 3, Day 4).

Concepts for posters depicted change through images that showed before- and after- scenarios to motivate action. It was also suggested that posters could be distributed for display within private homes to indicate support personalization an ownership of health concerns, rather than the only displaying such media at health facilities and other public venues.

\section{Discussion}

Malaria is a leading cause of illness and death among Around three out of ten children under the age of five $(29 \%)$ testing. Around two thirds of Malawing households $(63 \%)$ che at least one mosquito net, with more tha half of TNN owneship has increased over the past ten years (nathas been stable in the recent period ${ }^{8}$.

While global declines in malaria have been attributed to standardized control measures in combination with systematic scale-up of interventions in most-affected countries, there have been episodes of resurgence where evels of malaria incidence have rebounded, thereby raising questions about the sustainability of interventions. Factors been noted to include weakening of programmes through been the eduction of funding, down-scaling of indoor residual pristrecting in bed nets, and declines in community cooperation?. Engagement with community members through the Action Media process demonstrated that despite good knowledge of malaria (including adequate risk perception and strong compliance with LLIN use), malaria incidence was perceived
to be unacceptably high in the community. Concerns about to be unacceptably high in the community. Concerns about
continued exposure to mosquito bites were related to social action at community level, including holding meetings to pregnant women and children under the age of 5 in Malawi Demographic and Health Survey data collection, with $67 \%$ Demographic and Health Survey data collection, with $67 \%$ (ITN) concerns are not typically addressed through standardized approaches, and community members were frustrated by the contradiction between their commitment to LLIN use and their experiences of regular bouts of malaria. They were also worried about the ongoing negative consequences of illnes of their children and themselves that led to severe hardships and undermined family and community wellbeing.

While topical mosquito repellants are widely recommended for tourists and others travelling to malaria endemic areas, they have not been included among prevention strategies has been explored in various small-scale studies, primarily in Asia and South America, but also in a few African countries, and a review. Findings suggest minimal or no contribution to malaria control ${ }^{10,11}$, with constraints being largely related to inconsistent use of repellants ${ }^{12}$. Participants in the present study were strongly motivated to prevent malaria, and were of the view that they would make use of insect repellants to prevent mosquito bites prior to going to bed if these be strengthened through more effective community-driven communication, increasing potential for malaria prevention in such Malawian contexts.

Perspectives on the interaction between bed bug infestations and malaria control were revealing. Perceptions that LLINs infestation led some community members to that bed bug without protection from mosquitos undermined malaria prevention. Bed bugs are acknowledged globally to be difficult to control, and insecticide resistance constrains eradication efforts ${ }^{13,14}$. Some community members were saic to be boiling their LLINs to kill bed bugs, and this practice has potential to reduce effectiveness of the nets by causing anti-malarial insecticides to leach away prematurely. Given perceived high levels of malaria incidence in the community, that included severe illness and risk of death, participants were concerned that malaria treatment-seeking necessitated travel to distant health facilities. This was difficult for people who were seriously ill, and such visits also depleted household resources if transport costs were to be entertained. It thus made sense that provision of malaria treatment through local HSAs should be considered. Although the potential or need for rapid diagnostic tests was not raised by participants, atilization of rapid diagnostic tests do form part of Malawi's wervention strategy for the current period ${ }^{5}$. While there was some deference to the need for support to malaria example via external agencies or government - for upply of provision of chemicals to eradicate bed bugs noted that there were some steps that could be taken without outside intervention. Strategies mentioned included community management of potential mosquito breeding areas and moderating activities that increased exposure to mosquitos in the early evening. Overall, there was a strong inclination towards working collaboratively to address the malaria problem, and it was recognized that many actions were both simple and doable.

With regard to communication, the participatory process demonstrated the value of moving beyond literal and persuasive messaging towards conveying insights and
lessons through proverbs, songs and poems that could be 
presented by community members themselves. Proverbs offer greater opportunity to deepen meaning in support of health communication by connecting health concerns to cultural constructs and values. Interest in imagery that conveyed changes in preventive practices through before and after scenarios also supported motivation for change. The suggestion for expanding the distribution of health posters from public spaces to include the possibility of display in households indicates an interest in validation and ownership of health promotion within and by families. This suggests that communication based on values such as family responsibility, communality and taking action to improve health would have better traction than singular emphasis on persuasive communication conceived on a top-down basis that focuses on discrete behaviors.

\section{Conclusions}

Participatory research with health vulnerable community members highlights the relevance of additional prevention measures that are not typically considered as in conventional malaria prevention programming. While mosquito repellants do not appear to be effective as a complementary measure for malaria control in other settings, community members in this setting in Malawi appear to be intrinsically motivated to use mosquito repellant during critical times. Provision of treatment locally through HSAs would reduce time and costs of treatment seeking as well as potentially impacting malaria incidence. While it is unclear whether bed bugs are a widespread concern in Malawi, what this study reveals is that bed bug infestations have potential to considerably undermine the efficacy of LLINs, and measures to address this concern should be made available. The finding also highlights that formative and operational research should be undertaken to support efforts to eradicate malaria. Inclusion of community members in malaria prevention efforts should be prioritized, given that motivation to respond is prompted by a strong awareness of the counterproductive impacts of malaria on family and community life. Supporting indigenous formats of communication, including local idioms and cultural expression, increases opportunities for ownership of health response by community members.

\section{Abbreviations}

Insecticide Treated Net (ITN); Indoor Residual Spraying (IRS); Health Surveillance Assistant (HSA); Long Lasting Insecticidal Net (LLIN).

\section{Ethical considerations}

As this was formative research to inform programme design, it falls within the definition of non-research and ethical review was therefore not mandatory. Nonetheless, the study was guided by a formal research protocol that included an ethics component that was consistent with international guidelines.

\section{Acknowledgements}

We gratefully acknowledge the contribution of community members who participated in the Action Media sessions for this study, and members of the broader research team who assisted with fieldwork including the Ministry of Health, Health Education Section and organisations falling within the USAID Health Communication for Life (HC4L) programme. This study was funded through the generous support of the American people through the United States Agency for International Development (USAID). The contents are the responsibility of the authors and FHI360 and do not necessarily reflect the views of USAID or the United States Government. WP drafted the manuscript. TP and IK provided revisions to the manuscript. All authors were involved in the conception and design of the study and WP and IK were involved in data collection. All authors read and approved the final version of the manuscript.

\section{Competing interests}

The authors declare that they have no competing interests.

\section{References}

1. Glanz K, Bishop DB. The role of behavioral science theory in development and implementation of public health interventions. Annu Rev Publ Health. 2010;31: 399-418. doi: 10.1146/annurev. publhealth.012809.103604

2. Airhihenbuwa C, Obregon, R. A critical assessment of theories/ models used in health communication for HIV/AIDS. J Health Commun. 2000;1;5(S1):5-15. doi:10.1080/10810730050019528

3. Bandura A. Social cognitive theory of mass communication. Media Psychol. 2001;3(3):265-299. https://doi.org/10.1207/ S1532785XMEP0303_03

4. Dagron AG, Tufte T. Communication for social change anthology: Historical and contemporary readings. New Jersey: CFSC Consortium, Inc. 2006.

5. Morris, N. The diffusion and participatory models: a comparative analysis. In: O Hemer, T Eriksen. 2005. Media and glocal change: Rethinking communication for development. Buenos Aires: CLACSO. 2005.

6. Parker WM, Becker-Benton A. Experiences in conducting participatory communication research for HIV prevention globally: Translating critical dialog into action through action media. Front Public Health. 2016;4:1-10. doi: 10.3389/fpubh.2016.00128

7. GBD 2016 Causes of Death Collaborators. Global, regional, and national age-sex specific mortality for 264 causes of death, 1980-2016: a systematic analysis for the Global Burden of Disease Study 2016. Lancet, 2017;390:1151-1210. doi: 10.1016/S0140-6736(17)32152-9.

8. National Statistical Office Malawi and ICF. Malawi Demographic and Health Survey 2015-16. Zomba, Malawi, and Rockville, Maryland, USA: NSO and ICF. 2017

9. Cohen JM, Smith DL, Cotter C, Ward A, Yamey G, Sabot OJ, et al. Malaria resurgence: a systematic review and assessment of its causes. Malaria J. 2012;11(1):122. doi: doi.org/10.1186/1475-2875-11-122

10. Wilson AL, Chen-Hussey V, Logan JG, Lindsay SW. Are topical insect repellents effective against malaria in endemic populations? A systematic review and meta-analysis. Malar J. 2014;13;(1):446. doi: $10.1186 / 1475-2875-13-446$

11. Maia MF, Kliner M, Richardson M, Lengeler C, Moore SJ. Mosquito repellents for malaria prevention. The Cochrane Library. Issue 2. Art. No.: CD011595. DOI: 10.1002/14651858.CD011595.pub2. doi: 10.1002/14651858.CD011595.pub2

12. Moore, SJ. A new perspective on the application of mosquito repellents. Lancet Infect Dis. 2016; 16;(10):1093-1094. http://dx.doi. org/10.1016/s1473-3099(16)30207-9

13. Doggett SL, Dwyer DE, Peñas PF, Russell RC. Bed bugs: clinical relevance and control options. Clin Microbiol Rev. 2012;25(1)164192. doi: 10.1128/CMR.05015-11

14. Gordon, J R, Potter, M F, \& Haynes, K F. Insecticide resistance in the bed bug comes with a cost. Sci Rep. 2015;5. doi: 10.1038/srep10807

15. Malawi Ministry of Health. Malawi malaria communication strategy 2015-2020. Lilongwe: Ministry of Health. 2016. 